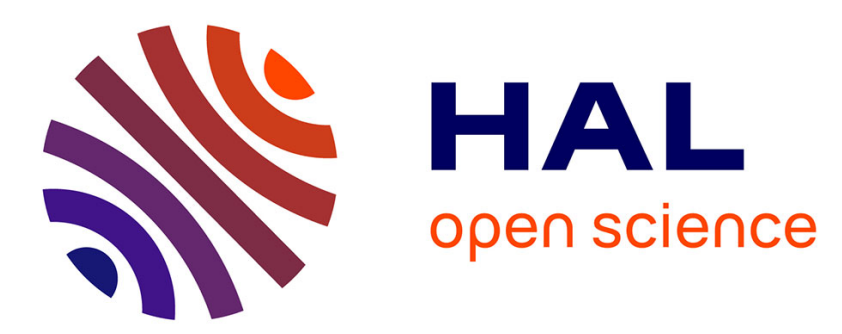

\title{
Energy Evaluation of PMCMTP for Large-Scale Wireless Sensor Networks
}

Jamila Ben Slimane, Ye-Qiong Song, Anis Koubâa, Mounir Frikha

\section{To cite this version:}

Jamila Ben Slimane, Ye-Qiong Song, Anis Koubâa, Mounir Frikha. Energy Evaluation of PMCMTP for Large-Scale Wireless Sensor Networks. The Fifth International Conference on Systems and Networks Communications, Aug 2010, Nice, France. pp.21-30, 10.1109/ICSNC.2010.10 . hal-00542255

\section{HAL Id: hal-00542255 \\ https://hal.science/hal-00542255}

Submitted on 3 Dec 2010

HAL is a multi-disciplinary open access archive for the deposit and dissemination of scientific research documents, whether they are published or not. The documents may come from teaching and research institutions in France or abroad, or from public or private research centers.
L'archive ouverte pluridisciplinaire HAL, est destinée au dépôt et à la diffusion de documents scientifiques de niveau recherche, publiés ou non, émanant des établissements d'enseignement et de recherche français ou étrangers, des laboratoires publics ou privés. 


\title{
Energy Evaluation of PMCMTP for Large-Scale Wireless Sensor Networks
}

\author{
Jamila Ben Slimane* ${ }^{*}$, Ye-Qiong Song ${ }^{\ddagger}$, Anis Koubâa ${ }^{\S \llbracket}$ and Mounir Frikha* \\ *Sup'Com-MEDIATRON, City of Communication Technologies, 2083 Ariana, Tunisia \\ ${ }^{\ddagger}$ LORIA and INPL, Campus Scientifique, BP 23954506 Vandoeuvre-les-Nancy, France \\ §IPP-HURRAY! Research Group, Polytechnic Institute of Porto, \\ Rua Antnio Bernardino de Almeida, 431, 4200-072 Porto, Portugal \\ ๑Al-Imam Muhammad ibn Saud University, Computer Science Dept., 11681 Riyadh, Saudi Arabia \\ Email: jamilabs07@yahoo.fr, Song@loria.fr, akoubaa@dei.isep.ipp.pt,m.frikha@supcom.rnu.tn
}

\begin{abstract}
One of the major concerns in wireless sensor networks (WSNs) is improving the network lifetime. This paper addresses the problem of energy saving in an IEEE 802.15.4a large-scale full mesh WSNs based on UWB technology. It presents a three-tiered network architecture to facilitate resource sharing and to ensure load and energy balancing. With a particular focus on medium access control (MAC) protocols, we propose a multi-channel MAC protocol, Prioritized Multi-Channel MultiTime slot MAC protocol (PMCMTP), that due to its intelligent management of spectrum resource and time slots, can simultaneously improve energy efficiency and network quality-of-service (QoS). To prove energy efficiency and QoS support of PMCMTP, we implement it in a discrete-time simulator built in JAVA. Simulations results show that PMCMTP succeeds to save energy and to enhance network QoS with a low overhead.
\end{abstract}

\section{Keywords-WHSNs; PMCMTP; energy.}

\section{INTRODUCTION}

Energy efficiency is the key issue for Wireless Sensor Networks (WSNs), which mainly rely on limited battery power supply. Indeed, energy is generally recognized as a key bottleneck for embedded sensor nodes. This bottleneck is exacerbated by the disparity between the rapidly growing processing speed and the slowly improving battery capacity of computing systems. Some solutions for saving energy at medium access control (MAC) layer for WSNs are put forward. The power wastage in WSNs, especially at MAC layer, is due mainly to: Idle listening, collisions, protocol overhead, overhearing and overemitting. Recently, several researchers have explored the possibility of using multiple channels to overcome the limitations of single channel MAC protocols in terms of QoS support (Real-time guarantee, throughput) and energy saving. Most commercial radio devices, such as MICAZ, TelosB, and CMU FireFly, already provide the basic functions required to support multiple channels, and as shown in [1] and [2], channel switch latency of the CC2420 transceiver is short (just about $200 \mu s)$.

In [3], we have proposed PMCMTP, a Prioritized MultiChannel Multi-Time slot media access control Protocol which is suitable for real-time and $\backslash$ or high data-rate applications. For more details about PMCMTP's performance, in terms of end-to-end delay guarantee and throughput, the reader can refer to [3]. In this paper, we focus on the evaluation of the PMCMTP's behavior in terms of energy saving. The main contributions of this paper are as follows:

- First, we investigate the advantages of UWB IEEE 802.15.4a [4] physical layer to minimize energy consumption by providing the first spectrum management scheme exclusively for full mesh large-scale WSNs.

- Second, we propose PMCMTP for dense and large-scale WSNs to ensure:

- An efficient resource allocation (channel frequencies and time slots inside each Personal Area Network (PAN)),

- Energy balancing and saving to prolong network lifetime,

- QoS support.

PMCMTP takes into account: the spatial channel reuse, the duty cycle's information of PANs and priorities of data stream. - Finally, we perform an evaluation of our protocol using simulations, demonstrating that it comes to reach our goals in terms of spectrum efficiency, energy efficiency and network performance enhancement.

\section{RELATED WORK}

In the literature, most proposed MAC protocols, focusing mainly on the problem of energy efficiency in WSNs, are based on the use of single channel, where a few multi-channel MAC protocols have been proposed for WSNs [5]-[12]. The first multi-channel protocol, called Multi-frequency Media access control for wireless Sensor Networks (MMSN) [5], represents four frequency assignment schemes for WSNs. Although MMSN achieves increased network throughput, the fixed channel allocations limit channel utilization. Moreover, it wastes a lot of energy due to several broadcasts and collisions. To overcome MMSN's deficiency, we propose (i) a dynamic channel allocation scheme based on network duty cycle's information and spatial channel reuse to enhance channel utility and (ii) a centralized multi-channel multi-time slot MAC protocol to ensure energy saving with a low overhead. YMAC [6] is a TDMA-based multi-channel MAC protocol for WSN. Y-MAC, based on scheduled access, assigns time slots to the receivers instead of the senders. At the beginning of each time slot, potential senders for the same receiver contend for the medium. We note that increased contention especially 
around the sink node with high data-rate scenarios can lead to bottleneck problem of the sink node. In this situation, several packets can exceed the delay bound of the underlying application and it can be dropped. So, neither QoS constraints will be respected nor energy will be saved. To avoid such situation, we propose to decompose our network on a set of PANs organized on cells to balance load and energy consumption in order to ensure network lifetime maximization and QoS support. To overcome the deficiency of single-channel LMAC protocol in dense networks, Multi-channel Lightweight Medium Access Control (MLMAC) [7] has been proposed. In single-channel LMAC, the number of transmissions is limited by the number of time slots in a frame. However, in MCLMAC time slots are selected with frequencies. Although, MC-LMAC exhibits better performance than LMAC in terms of throughput due to parallel transmissions, energy saving is not improved. Also, collisions can occur when network topology changes which leads to energy wastage. For that, we propose a centralized multi-channel MAC protocol insensitive to the network topology change. In [8] and [9], the authors proposed a dynamic channel allocation based on agreement established between each sender and receiver nodes. Such approach may be suitable in light network but in dense network frequency negotiation messages can involve a considerable unnecessary overhead. The advantage of those protocols is the use of several channels for control traffic which can avoid control channel congestion problem. There are also efforts in industry which utilize multi-channel radios. Time Synchronized Mesh Protocol (TSMP) [10] is a TDMA-based frequency hopping networking protocol for wireless mesh sensor networks (WMSNs). TSMP maintains synchronization among nodes. Nodes employ frequency hopping according to a shared pseudo-random schedule. All previous multi-channel allocation schemes are proposed for classical WSNs operating on 2.4 Ghz band without any support of QoS mechanisms, however the authors in [11] proposed the first Multi-Channel MAC protocol (MCMAC) taking into account the notion of priority during channel allocation process inside a cluster. This protocol is based on four stages: synchronous beacon, transmission request, channel schedule and data convey. Although MCMAC ensures multi-channel access in cluster tree WSNs but it does not support the simultaneous communications of several clusters. Moreover, the cluster header assigns channels to its members for a fixed duration which leads to the wasting of resource (if this duration is bigger than needed duration) and $\backslash$ or to the increase of communication overhead (if this duration is shorter than needed duration). In [12], the authors proposed the first multi-channel scheme designed for UWB based IEEE 802.15.3 networks. Based on dynamic traffic demand, the proposed mechanism employs a distributed dynamic channel allocation algorithm (DCA) [13] to distribute the channels among neighboring piconets. Because of several broadcasts (between piconet controllers), the communication overhead in this mechanism is relatively high.

Similar to [12], we propose to organize the global network on set of PANs, to reduce the complexity of resource sharing.
But, for channel and time slot allocation in UWB based WSNs, we propose a mechanism that meets WSNs requirements and ensures QoS support in such networks. To prolong network lifetime and to support QoS, we propose to ensure energy efficiency and network performance enhancement (throughput, delay and QoS support) by exploiting the following key ideas:

1) Advantages offered by UWB technology: low power transmission, immunity to multi-path propagation, high data-rates and high-precision ranging capability,

2) Adequate network architecture that can reduce the complexity of the resource sharing task in dense and largescale full mesh WSNs and balance load and energy.

3) Multi-channel multi-time slot access protocol ensuring parallel transmissions and congestion avoidance to:

- shorten active period (extend sleep period as possible) that leads to energy saving and network lifetime maximization,

- increase throughput and decrease delay.

4) Support of data stream prioritization to ensure QoS.

The rest of the paper is organized as follows: In Section 2, we present the system model. Section 3 gives an overview of the PMCMTP protocol. Section 4 details power consumption in WSNs. In Section 5, we evaluate PMCMTP performance by analyzing and commenting some simulation results.

\section{SYSTEM MODEL}

In order to deploy a dense network supporting a considerable number of nodes, we proposed in [14] a three-tiered network to represent the global network, using UWB sensors in the first and second network levels. The choice of the UWB technology is done to benefit from:

- Power efficiency: its extremely low transmitting power minimizing interference,

- Real-time guarantees: high data-rates allowing real-time and high data-rate applications,

- Location awareness: location capacity ensuring mobility management and node identification.

For the third tier, we proposed to use WiFi to benefit from its high data-rate, large coverage and security aspects. We aim to design a Wireless Hospital Sensor Network (WHSN) for an application in a hospital, but the proposed system model as well as channel allocation schemes can apply to more general WSNs contexts. Figure 1 shows all WHSN's layers:

- The Body Sensor Network (BSN) tier: The lowest level represents the Body Sensor Network (BSN). We can model an elementary BSN by a star network composed of one coordinator and a set of biosensors that ensure physiological measurements and patient's medical monitoring of patient.

- The Personal Area Network (PAN) tier: To improve network performance in a dense hospital environment, we propose overlaying the network of BSNs with a second upper level network or PAN. As shown in Figure 1, the network is represented by a hexagonal cell of sensors organized in mesh topology including one PAN coordinator, 
several routers (which relay sensing information toward PAN coordinator and can execute some sensing measurements such as humidity, temperature measurements, etc.) and and several mobile BSNs (only BSN's coordinator communicates directly with its PAN coordinator and router nodes).

- The UWB/WiFi based Mesh Network tier: For an efficient solution for channel allocation and mobility management in WHSNs, that cellular architecture, based on UW$\mathrm{B} / \mathrm{WiFi}$ technologies, is chosen to the third level to have at the end a three-tier hierarchical cellular network.

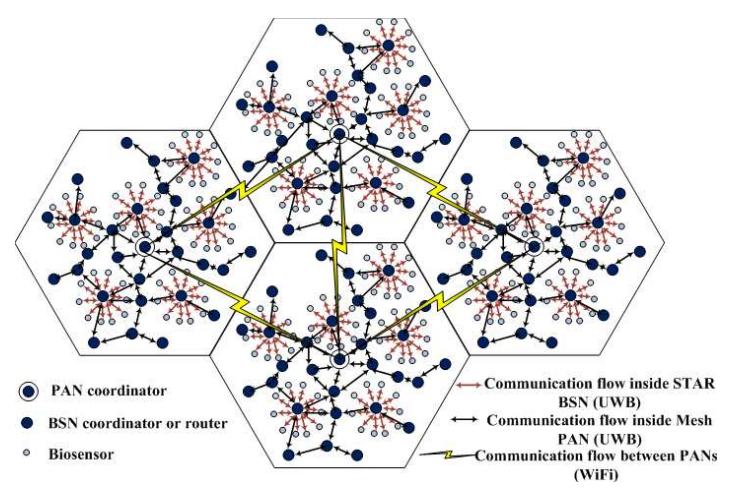

Fig. 1. WHSN architecture

Inside a PAN, only BSNs are mobile with very low rate. The study of mobility is out of this paper. The detailed description of the network architecture is out of the scope of this paper, so for more details, the reader can refer to [14]. Let us assume the general case of a network composed by a set of PANs uniformly distributed. We define $N_{c}$ as the number of PANs. The ideal case of an hexagonal model is chosen to ensure the total coverage of the network. Although the coverage zone of a sensor device is not an hexagon or a perfect circle in practice, there are procedures and mechanisms [15] that ensure the adjustments of the model during network deployment by means of experimental tests and measurements.

The IEEE 802.15.4a Impulse-Radio (IR) UWB complaint devices can operate in three independent bands: (i) the subgigahertz band (250-750 MHz), (ii) the low band (3.1-5 GHz) and (iii) the high band (6-10.6 GHz) (See Figure 2). As shown in the table $39 \mathrm{~d}$ given in [4], the standard specifies 16 physical frequency channels associated with 8 sequence codes to have in total 32 logical channels.

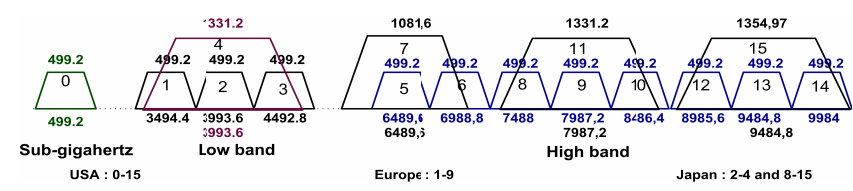

Fig. 2. IEEE 802.15.4a UWB plan bands

The IEEE 802.15.4a MAC protocol supports two operational modes that can be selected by the PAN Coordina- tor: Beacon-enabled mode and non beacon-enabled mode. To provide time guarantees to deliver data frames, beaconenabled mode is used. The format of the superframe is defined by the PAN Coordinator. The superframe, corresponding to the Beacon Interval $(B I)$, is defined by the time between two consecutive beacons, and includes an active period and, optionally, a following inactive period (See Figure 3(a)). The active period, corresponding to the Superframe Duration $(S D)$, is divided into 16 equally sized time slots, during which data transmission is allowed. For a global network of $N_{c}$ PANs, each PAN coordinator is characterized by its superframe duration $\left\{P A N_{i}=\left(S D_{i}, B I_{i}\right)\right\}_{1<i<N_{c}}$ as shown in Figure 3(b). We define $\overline{B I_{\operatorname{maj}}}$ and $\overline{S D_{\min }}$ as respectively the major cycle (the least common multiple of all PANs $B I$ ) and the elementary active cycle (the least common denominator of all PANs $S D$ ). To efficiently schedule the $S D_{i}$ durations, we can use the methods proposed in [16], [17].

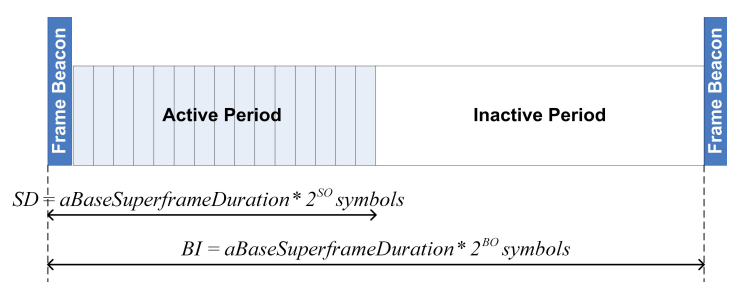

(a) PAN superframe structure

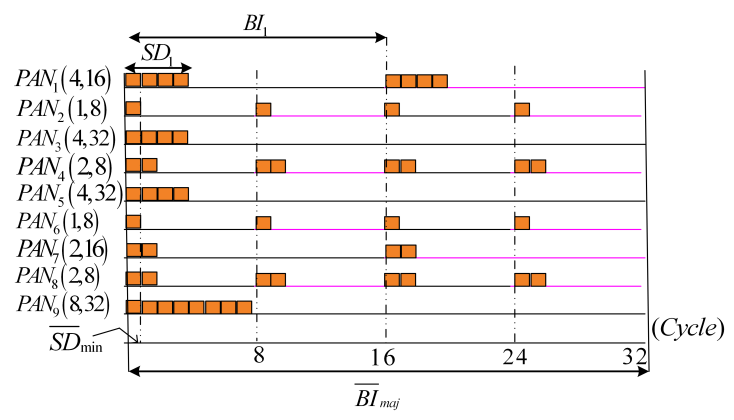

(b) Network duty cycle

Fig. 3. Network configuration

\section{PMCMTP FOR IR UWB SENSOR NETWORKS}

The role of WHSN is to ensure real-time and continuous patient monitoring to reduce time of routine consultation and to immediately treat emergency cases. So, such network must support a large number of BSNs or patients with different states, which must be monitored by means of various types of biosensors. Consequently, the network must present a long lifetime and support QoS ensuring efficient patient monitoring.

In order to balance energy consumption, maximize the network lifetime and enhance such QoS (in terms of network capacity or throughput, delays, prioritized physiological measurements support), we propose a MAC protocol called PMCMTP for mesh WSNs taking into account:

- Full mesh topology with multi-hop routing to ensure load and energy balancing, 
- Spatial channel reuse in order to efficiently assign several channels per PAN without suffering from co-channel interference,

- PANs duty cycle in order to dynamically allocate channels, maximize channel utility and avoid idle listening,

- Data stream prioritization at the level of PANs and BSNs, to ensure QoS support per patient and per service.

\section{A. First Level of Channels Allocation}

Inside a WSN, we distinguish two types of traffic: Control and data communication traffics. Control traffic is generated to identify each PAN, to synchronize devices that are associated with a PAN and to manage communication between each PAN's members. Data communication traffic represents the useful information to be transmitted between devices. In [18], we have proposed an allocation strategy for control and data channels.

1) Control channel allocation: To avoid control channel congestion [8], we propose to statically assign one control channel to each PAN. The emission power density of the UWB signals is less than $-41.3 \mathrm{dBm} / \mathrm{MHz}$ [4]. Given that, overlapping channels $(4,7,11$ and 15) are characterized by high bandwidth (more than $1 \mathrm{GHz}$, see Figure 2), they can allow higher transmit power (the transmit power limit varies from $80.1 \mu W$ to $100.4 \mu W$ ), permitting an extended range, as compared to non-overlapping channels (with $499.2 \mathrm{MHz}$ of bandwidth allowing a transmit power limit of $37 \mu W$ ). So, to persistently cover each cell with control traffic, we find that the overlapping channels are more suitable to ensure the zone coverage for such traffic. An optimal coloring algorithm is used to share control channels between PANs without suffering from inter-PAN interference.

2) Dynamic data communication channel allocation: According to network configuration and by means of an optimal coloring algorithm, we propose to allocate the set of residual channels (non-overlapping channels and the supplementary overlapping channels with their appropriate sequence codes) for data communication. According to PAN's duty cycle and available channel frequencies, each active PAN coordinator can benefit simultaneously from several data communication channels during each elementary active cycle $\overline{S D}_{\min }$.

The detailed description of the Ultra Wide Band Channel Allocation Scheme (UWBCAS), for control and data channels sharing between PANs, is out of the scope of this paper, so for more details, the reader can refer to [18].

\section{B. Second Level of Data Channels Allocation (inside a PAN)}

In this subsection, we present PMCMTP [3] for logical channels and time slots allocation inside each PAN. Similar to [19], a key concept in PMCMTP is the elementary active cycle, which is composed by two consecutive active periods, the first for synchronization and collect of resource requests, and the second for the Request Scheduling Algorithm (RSA) process, reception of second beacon and allowable data communications (See Figure 4).
For each elementary active cycle, the PAN coordinator collects all the resource allocation requests of its network's members. Then, according to the spectrum sharing scheme [18], it can know the number of channels that it can benefit from during the current active cycle. Next, it tries to allocate available time slots per channel in response to collected requests. Finally, concerned sensors can begin their data communications. The principle of PMCMTP is based on the three following phases:

1) Time Slots Request phase: Transmission requests phase must precede each PAN's active period. This phase is divided into two sub-steps: (i) the first step consists in PAN synchronization, (ii) the second step consists in collecting all transmission requests from PAN's members. During this phase, the allocated control channel is used. During the first step, by listening to the beacon frame, PAN members adjust their wake-up clocks. The second step represents a set of equal short time slots, during which, the PAN coordinator is listening to the requests of PAN's member. So, just following the reception of the first beacon frame, each PAN's member waits for its own time slot in order to send its request packets.

2) Channels/time slots allocation phase: According to the $R S A$ algorithm, after reception of all transmission requests, the PAN coordinator tries to schedule it according to its assigned priority. Once the list of requests are scheduled, the PAN coordinator tries to launch the phase of time slots and channels allocation. For each request, it tries to find the earliest available time slots per channel to assign it to the suitable request. At the end of the process of time slots and channels allocation, PAN coordinator registers a trace of requests, which were not served in its queue in order to analyze it during the next cycle. Then, it inserts into the next beacon frame the necessary information (Request identifier, index of allocated channel, index of the first allocated time slot, number of allocated time slots, address of the request's sender and of flow destination) of the served requests.

3) Data transmission phase: After listening to the second beacon frame, PAN's members can have a feedback of their transmission requests. Each concerned sensor switches to the suitable channel at the suitable time slot and it begins sending or receiving data frames during the reserved duration.

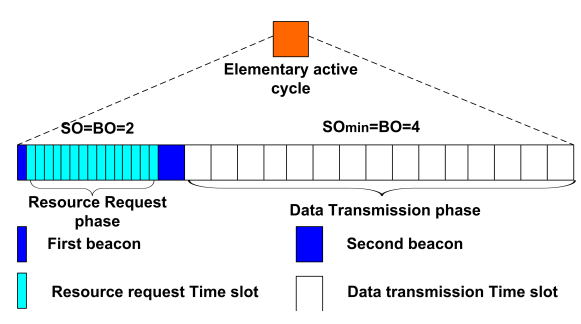

Fig. 4. An elementary active cycle

The detailed description of PMCMTP is out of the scope 
of this paper, so for more details, the reader can refer to [3].

\section{ENERGY EVALUATION METRICS IN WSNS}

In WSNs, all protocol layers consume energy. We know that WSNs are power-constrained since nodes operate with limited battery energy. So, in order to save energy, maximize the lifetime and the power efficiency of the global network, all network protocol layers must cooperate to ensure an efficient energy management.

\section{A. Energy Consumption}

Let $E_{\text {Total }}$ represents the sum of the energy consumed by all network's members. For each node, the energy consumption is the sum of energy consumed during different power states [20]:

- Energy consumed during Radio transmission, $E_{T X}$;

- Energy consumed during Radio receiving, $E_{R X}$;

- Energy for circuit operation during idle state, $E_{I D L E}$;

- Energy consumed at sleep state, $E_{S L E E P}$;

Therefore, suppose that the power consumption in transmitting, receiving, idle and sleep states are respectively $P_{T X}$, $P_{R X}, P_{I D L E}$ and $P_{S L E E P}$. Let $E_{i}^{j}$ represents the energy consumption of the $i^{\text {th }}$ node of the $j^{\text {th }}$ PAN:

$$
\begin{array}{cc}
E_{i}^{j}= & E_{T X_{i}^{j}}+E_{R X_{i}^{j}}+E_{I D L E_{i}^{j}}+E_{S L E E P_{i}^{j}} \\
E_{i}^{j}= & P_{T X} \times T_{T X_{i}^{j}}+P_{R X} \times T_{R X_{i}^{j}}+ \\
& P_{I D L E} \times T_{I D L E_{i}^{j}}+P_{S L E E P} \times T_{S L E E P_{i}^{j}}
\end{array}
$$

where $T_{T X_{i}^{j}}^{j}, T_{R X_{i}^{j}}^{j}, T_{I D L E_{i}^{j}}$ and $T_{S L E E P_{i}^{j}}$ represent time durations spent by the $i^{\text {th }}$ node of the $j^{\text {th }}$ PAN during respectively transmitting, receiving, idle and sleep states. We assume that PAN coordinators are mains powered, but the energy consumption of the members of each PAN (except the PAN coordinator) must be well-managed to prolong the network lifetime. The energy consumptions of respectively the $j^{\text {th }}$ PAN and the global network are given by $E^{j}$ and $E_{\text {Total }}$. The average energy consumption $\overline{E_{\text {node }}}$ is obtained by averaging the energy consumption of all nodes.

$$
E^{j}=\sum_{i=1}^{N_{j}} E_{i}^{j}, E_{\text {Total }}=\sum_{j=1}^{N_{c}} E^{j}, \overline{E_{\text {node }}}=\frac{E_{\text {Total }}}{\sum_{j=1}^{N_{c}} N_{j}}
$$

where $N_{c}$ and $N_{j}$ represent respectively the total number of PANs and the number of the $j^{\text {th }}$ PAN's members.

In the literature, several UWB transceivers have been proposed [21]-[26]. The power consumption of UWB transmitter varies from $2 \mathrm{~mW}$ to $15 \mathrm{~mW}$ and that of UWB receiver varies from $21.6 \mathrm{~mW}$ to $50.1 \mathrm{~mW}$. Table I shows the default value of the power consumption of a UWB device and a classical device for different states. For idle and sleep modes, we admit the same power consumption for both devices (there is not any information about power consumption of idle and sleep modes of UWB devices).
TABLE I

POWER CONSUMPTION OF DIFFERENT STATES OF NODE

\begin{tabular}{|c||c|c|}
\hline \multicolumn{1}{|c||}{ Parameter } & \multicolumn{3}{c|}{ Default value of Power Consumption } \\
& UWB Transceiver [21] & CC2420 Transceiver [2] \\
\hline \hline$P_{I D L E}$ & - & $0.766 \mathrm{~mW}$ \\
\hline$P_{S L E E P}$ & - & $36 \mu W$ \\
\hline Max $P_{T X}$ & $12.6 \mathrm{~mW}$ & $31.32 \mathrm{~mW}$ \\
\hline$P_{R X}$ & $28.8 \mathrm{~mW}$ & $35.47 \mathrm{~mW}$ \\
\hline \hline Power supply & \multicolumn{2}{|c|}{$1.8 \mathrm{~V}$} \\
\hline
\end{tabular}

IDLE mode: crystal oscillator and voltage regulator $O N$

$S L E E P$ mode: only volatge regulator $O N\left(\right.$ ie mote $c^{\prime} n^{\prime} t$ hear the radio), Max $P_{T X}$ : calculated according to the maximum transmit power.

Figure 5 presents the IEEE 802.15.4a's data frame structure, where $S H R, P H R, P S D U, S I F S$ and LIFS refer respectively to synchronization header, physical layer header, physical layer service data unit, short interframe spacing and long interframe spacing.

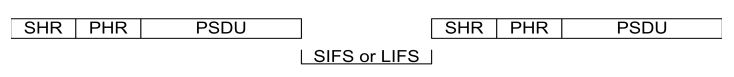

Fig. 5. IEEE 802.15.4a's data frame structure

Table II shows the default timing value of the IEEE 802.15.4a's data frame fields.

TABLE II

IEEE 802.15.4A's DATA Frame DuRATION

\begin{tabular}{|c||c|}
\hline Parameter & Default value \\
\hline \hline SHR preamble & $71.5 \mu \mathrm{s}$ \\
\hline PHR field & $16.4 \mu \mathrm{s}$ \\
\hline DATA field & $\frac{L_{\text {data }}}{\text { Rate }}$ \\
\hline SIFS/LIFS & $\frac{24}{\text { Rate }} / \frac{80}{\text { Rate }}$ \\
\hline
\end{tabular}

$L_{\text {data }}:$ Data length in bits,

Rate: Data-rate which can vary from $110 \mathrm{Kbps}$ to $27.24 \mathrm{Mbps}$

\section{B. Metrics}

In our evaluation, we will consider two metrics:

1) Network lifetime: The main metric of performance is the lifetime of the global network. This metric can be defined by various manners:

- Time of the first node failure [27] (TFF): defined as the time till the first node, in the network, runs out of battery energy.

- Fraction of surviving nodes in a network [28] $\left(F_{s}(t)\right)$ : defined as the sum of surviving nodes at time $\mathrm{t}\left(N_{s}(t)\right)$ per the number of total network members $\left(N_{\text {Total }}\right)$ :

$$
F_{s}(t)=\frac{N_{s}(t)}{N_{\text {Total }}}
$$

- Time of the last node failure [29] or Max expiration time $(M E T)$ : defined as the time till the last node, in the network, runs out of battery energy.

2) Power efficiency: Power efficiency [30] $P_{\text {eff }}$ is defined as the throughput achieved per unit of energy consumed, where the throughput represents the number of successfully delivered packets.

$$
P_{\text {eff }}=\frac{\text { Throughput }(\text { Packets })}{E_{\text {Total }}(\text { Joules })}
$$




\section{PERFORMANCE EVALUATION}

We have implemented PMCMTP in a custom WSN simulator built in JAVA [31] and based on some functionalities defined by Prowler simulator [32] with the support of the three-tiered network architecture proposed in [14] and the UWB channel allocation scheme detailed in [18]. It provides a nice graphical user interface to facilite the network configuration and to easily customize application scenarios that we propose to simulate. In this section, we propose to evaluate the performance of PMCMTP with default value of the network's parameters and then by varying the following parameters: channel number, system load, packet length, data-rate and node number.

\section{A. Simulation Parameters}

Let us consider a synchronized network of 9 PANs, each occupies hexagonal cell of radius $R=5 \mathrm{~m}$. Each PAN has 25 nodes uniformly distributed (One PAN coordinator, twenty routers and four BSNs coordinators). For each active PAN, we assume that nine nodes initiate gossip CBR streams towards the sink nodes and each source node generates a packet in every time slot. Each PAN coordinator is characterized by its superframe duration $\left\{P A N_{i}=\left(S D_{i}, B I_{i}\right)\right\}_{1 \leq i \leq 9}$ as shown in Figure 3(a) and Figure 3(b). Table III shows the default value of each parameter in the simulations. To eliminate the bottleneck problem of single sink node, we assume that there are several sink nodes in each PAN.

TABLE III

SIMULATION PARAMETER

\begin{tabular}{|c|c|}
\hline Parameter & Default value \\
\hline Number of PANs & 9, See Figure 3(b) \\
\hline PAN's radius & $5 m$ \\
\hline Number of Nodes per PAN & 25 \\
\hline Node placement & Uniform \\
\hline Communication rate & 850 Kbps \\
\hline System Load & 9 packets per PAN per Time slot \\
\hline Traffic pattern & Gossip CBR Streams \\
\hline Radio range & $5 m$ for control, $2 m$ for data \\
\hline Max MSDU length & 85 Bytes \\
\hline Time slot duration for data transfer & $0.984 \mathrm{~ms}$ \\
\hline Time slot duration for resource request & $0.246 \mathrm{~ms}$ \\
\hline$S O_{\min }$ for data communications & 4 \\
\hline Network Duty Cycle & See Figure 3(b) \\
\hline
\end{tabular}

\section{B. Evaluation of Energy Consumption}

1) The basic scenario: The simulation of the basic scenario (using the default value of each parameter as given in Table III) gives an idea on the behavior of the PMCMTP protocol according to time progress. As presented in Figure 6, the red curve shows the energy consumed by one PAN during the first elementary cycle, and the blue curve gives a global vision of energy consumption of the entire network during the first elementary cycle where all PANs are active. According to Figure 6, we note that energy consumed during synchronization and resource request phases, including the first and second beacons, is negligible compared to energy consumed during data transfer. Due to the extremely short length of request messages that energy consumption during synchronization and resource request is very low, it represents $6.5 \%$ of energy consumed during data transfer phase. Thanks to its low overhead and simplicity, this protocol can greatly improve the energy efficiency of PAN devices. Figure 7 gives a global vision of energy consumption of the first PAN and the global network during a major cycle. According to the red curve, we note that the energy consumed during sleep periods is extremely low (of a few $\mu J$ ) where the energy consumption of active period follows an increasing curve of almost linear trend. We can explain the linear trend by the use of CBR streams. According to the blue curve, we note that the energy consumed is a function of the number of active PANs. More the number of active PANs increases more the rate of energy consumption grows.

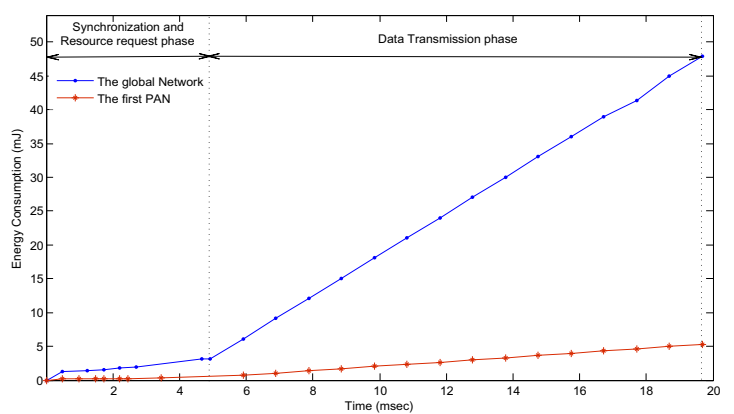

Fig. 6. Energy consumption of the global network and the first PAN during the first elementary cycle

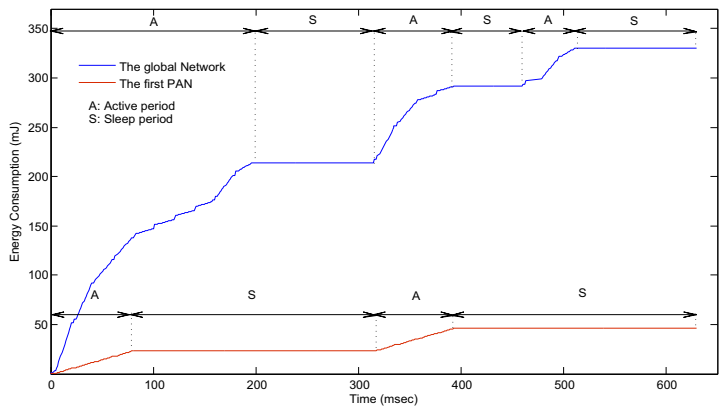

Fig. 7. The energy consumption's behavior of the global network and the first PAN

2) Energy Consumption vs Number of Channels : By varying the number of channels assigned to each PAN, we take the total of the consumed energy as a metric to measure our protocol performance. In this simulation, we conduct experiment with a load of 135 packets per PAN per elementary active cycle (i.e., 9 packets per time slot per active PAN). Figure 8 shows that our protocol becomes more energy efficient when we increase the number of channels. We see that the energy consumption decreases by $25.56 \%$ when the number of channels increases from 1 to 9 . According to Figure 8, the speed of energy consumption decrease slows down when 
the number of channels increases more. We think that the proportion of energy spent on the control overhead becomes little when increasing the number of channels (with more channels, the active periods become shorter so less control traffic). To avoid supplementary cycles (extra overhead) and the energy waste, it will be suitable to dispose of the necessary number of channels ensuring the processing of network's load during the shortest period. For this reason, we proposed in [18] a channel allocation scheme ensuring static control channel allocation and dynamic channel allocation (based on the spatial channel reuse and the duty cycle's information of PANs) in order:

- To increase the number of simultaneous communications, which leads to delays reduction, throughput increasing and energy saving,

- To avoid the phenomena of congestion (data and control traffic) and reduce energy waste.

In this way, we can meet concurrent constraints of energy and QoS. Moreover, we note a constant behavior of energy consumption when the number of used channels exceeds nine. We can explain this by the fact that the number of available channels exceeds the number of needed channels to support all simultaneous streams (i.e., 9 CBR per active PAN). So, in this case, the supplementary channels, exceeding the necessary number of channels, are not used, what leads to spectrum resource inefficiency. For a trade-off between resource utility enhancement, QoS guarantees and energy saving, we must define the rational duty cycle for each PAN according to the supported load.

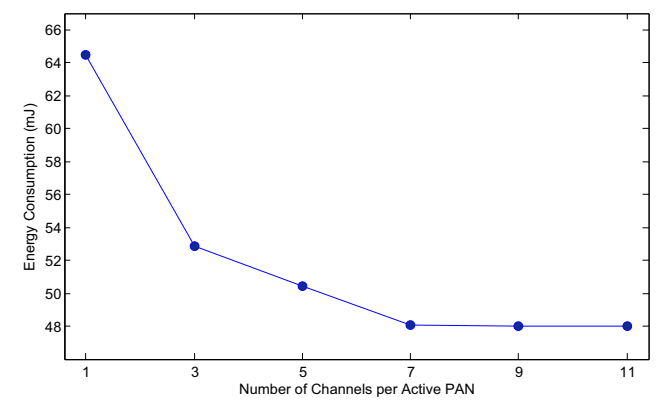

Fig. 8. Energy Consumption of the entire network vs Number of Channels

3) Energy Consumption vs System Loads: In this part, we explore PMCMTP's performance when different system loads are used, which are generated by different numbers of CBR streams. To analyze performance scalability, we conduct all experiments with different packet sizes. We distinguish two sets of experiments:

- The first set of experiments is conducted for a fixed duration of a major cycle (Figure 9.a),

- The second set of experiments is conducted for a fixed amount of load of 135 packets per PAN (Figure 9.b).

As Figure 9.a shows, for all the system loads varying from $9 \mathrm{CBR}$ streams to $81 \mathrm{CBR}$ streams (i.e., $1 \mathrm{CBR}$ per
PAN to 9 CBR per PAN), it is observed that PMCMTP always exhibits better performance when shortest packets are used because for a fixed duration that leads to minimum load and then to minimum energy consumption. According to Figure 9.b, it is observed that PMCMTP always exhibits better performance when largest packets are used because for a fixed amount of load that leads to shortest active period and then to minimum energy consumption. According to the application's QoS requirements (end-to-end delay guarantee, etc.), physical link quality and the rate of residual energy per node, that the suitable packet length can be defined.

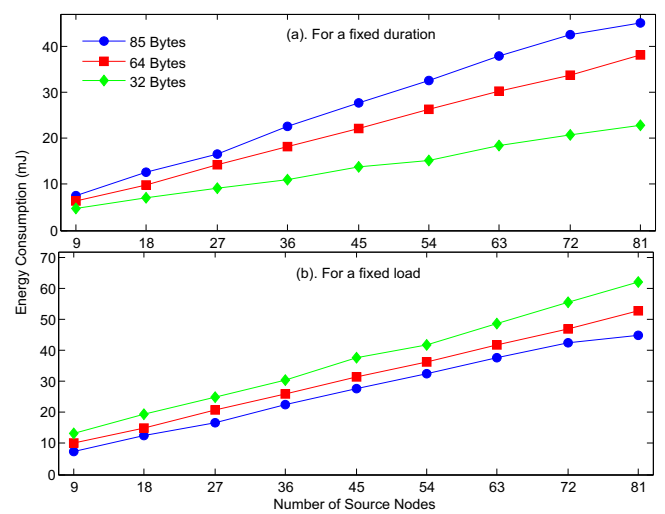

Fig. 9. Energy Consumption of the entire network vs Number of Source Nodes

4) Energy Consumption vs Number of Nodes: Figure 10 highlights the behavior of PMCMTP in terms of total energy consumption by varying the number of nodes. In this simulation, we conduct experiments with a duration of one elementary cycle and with the same system load (9 CBR per active PAN). We note that the behavior of PMCMTP, in terms of energy consumption, is almost invariable to the variation in the number of nodes. Given the fair time slot allocation method proposed by PMCMTP, idle listening is avoided and energy is mainly consumed during effective transmission and reception phases. Moreover, the proportion of energy spent during request phase (without considering synchronization phase) and transmission phase is almost the same with different number of nodes.

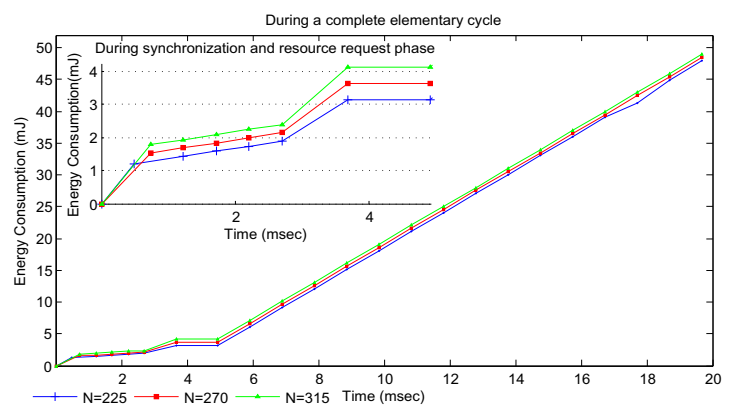

Fig. 10. Energy Consumption of the entire network vs Number of Nodes 
Although the slight impact of the variation of the number of nodes on PMCMTP's energy consumption behavior, we propose to analyze the energy consumption behavior of PMCMTP during synchronization and resource request phase as a function of node number (See Figure 10). We note that the energy consumption slightly increases with the increase of the number of nodes, this is due to the increase of the amount of energy spent in beacon frames reception.

5) Energy Consumption vs Data-rate: To analyze the effect of the increase of data-rate on our protocol's performance, we conduct simulation with different data-rates proposed by the IEEE 802.15.4a standard. Figure 11 shows that, PMCMTP becomes more energy efficient when data-rate increases. So, according to physical channel's state, we propose to implement a data-rate control policy ensuring data-rate selection in order to save energy, shorten delays and increase throughput as well.

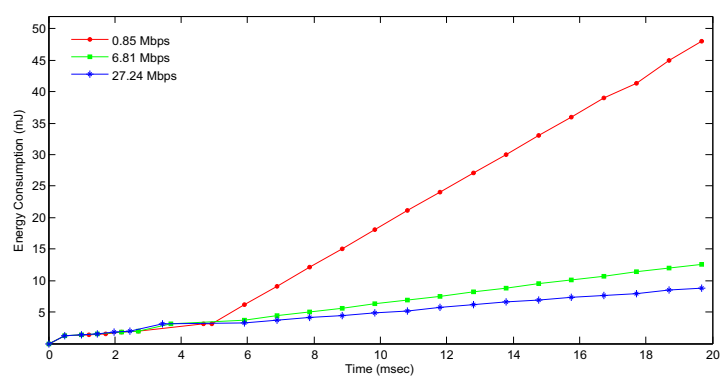

Fig. 11. Energy Consumption of the entire network vs Data-rate

\section{Comparative Performance Evaluation}

In this part, we compare the PMCMTP's performances with different physical layers (UWB IEEE 802.15.4a and IEEE 802.15.4 physical layers) and to MCMAC [11] protocol.

1) Energy Consumption: Taking the average energy consumption per node and/or the total energy consumption of the entire network as metrics, we propose to compare the PMCMTP's behavior on the one hand with both IEEE802.15.4 and IEEE802.15.4a physical layers, and on the other hand to the MCMAC's behavior.

- UWB IEEE 802.15.4a vs IEEE 802.15.4: In the following simulation, we conducted experiments, for a duration of a major cycle, with a load of nine CBR streams per active PAN. As shown by Figure 12, the difference of energy consumption between both cases (UWB and 2.4 physical layers) grows quickly during active periods to reach a factor of 3.71 at the end of cycle. We can explain that by the extremely low transmitting and receiving powers of a UWB IEEE 802.15.4a transceiver compared to a IEEE 802.15.4 transceiver. Moreover, high datarate offered by UWB physical layer can reduce delays (including delays of transmission and reception) which leads to energy consumption decrease.

- PMCMTP vs MCMAC: In this part, we conducted experiments, for a duration of an elementary cycle, to highlight and compare the energy consumption behaviors of PMCMTP and MCMAC protocols by varying one parameter (Number of nodes, number of CBR streams per active PAN, number of used channels per active PAN). In all experiments, as shown in Figure 13, PMCMTP offers better results than MCMAC.

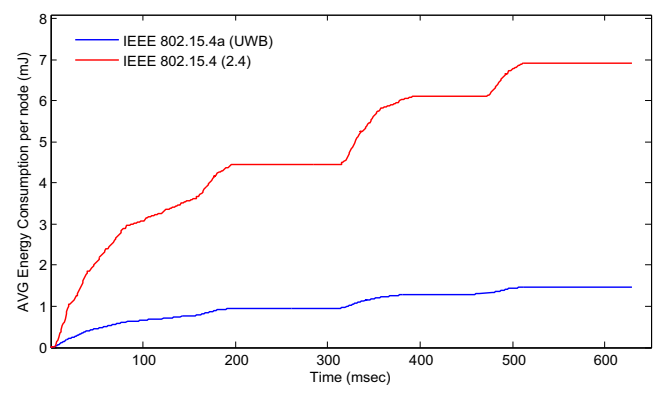

Fig. 12. Energy Consumption vs Physical layers

As shown by Figure 13.(a), the energy consumption for both protocols grows slowly with node number increase. We can explain this by the use of a fixed system load for all simulation experiments and given that the majority of energy consumption is due to data transfer and the amount of energy consumed by added nodes is small (just for receiving beacons and sleeping). But we note that MCMAC's energy consumption represents 3.5 times of that of PMCMTP's given that the MCMAC allocates data channels for a fixed duration (one time slot), which introduces additional communication overhead (supplementary requesting time slot phases).
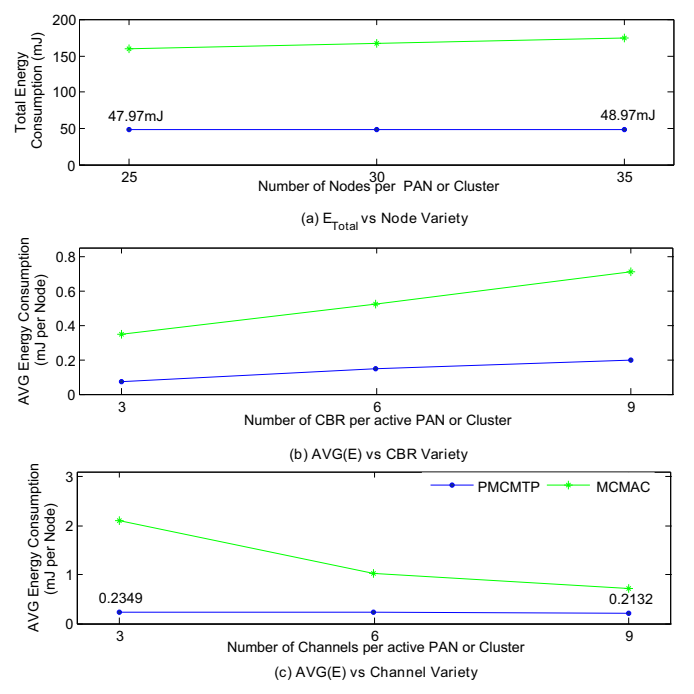

Fig. 13. PMCMTP vs MCMAC

By increasing the number of CBR streams, the difference between PMCMTP and MCMAC increases by $186 \%$ when the system load increases from 3 to 9 streams (See Figure 13.(b)). This difference is due to the additional communication overhead which increases with the increase of the number of CBR streams in the case of MCMAC. 
Figure 13.(c) shows that the difference of energy consumption between both protocols decreases by $26.67 \%$ when the number of used channels per active PAN (or Cluster for MCMAC) increases from 3 to 9. So, the increase of the number of used channels ensures the shorten of the active periods and the avoidance of supplementary communication overhead which leads to the energy consumption reduction for both protocols.

2) Network lifetime: Taking the network lifetime (as defined in subsection V-B1) as a metric, we evaluate the performance of PMCMTP protocol. The Initial Energy $(I E)$ represents the initial amount of battery energy before any activity. The relation between battery voltage $(U[V])$, battery capacity $(C[m A h])$ and initial energy $(I E[$ Joule $])$ :

$$
I E=U[V] \times C[m A h]=[\text { Watt } \times \text { sec }]=[\text { Joule }]
$$

We assume the use of one AA Battery (1.8V, 2500mAh), so, the initial energy is about 16200 Joule. Moreover, we assume a periodic network duty cycle (The repetition in the time of the network duty cycle illustrated in Figure 3(b)), in this case, we can estimate $T F F, F_{s}(T F F)$ and $M E T$ by:

$$
\begin{aligned}
& \widehat{T F F}=\frac{I E \times D_{\text {simulation }}}{\max \left(E_{\text {node }}\right)} \\
& F_{s}(\widehat{T F F})=\frac{\begin{array}{c}
N_{\text {Total }}-\mid\{\text { node } \\
\left.E_{\text {node }}=\max \left(E_{\text {node }}\right)\right\}\left.\right|_{t=D_{\text {simulation }}} ^{\text {node } \in \text { network }}
\end{array}}{\widehat{M E T}=\frac{I E \times D_{\text {simulation }}}{\min \left(E_{\text {node }}\right)}} \\
& D_{\text {simulation }}=k \times D_{\text {major cycle }}, k \in \mathbb{N}^{*}, \\
& D_{\text {major cycle }}: \text { Duration of a major cycle. } \\
& E_{\text {node }}: \text { Energy consumption of a node } \in \text { network. }
\end{aligned}
$$

Because of several simultaneous transmissions, at $\widehat{T F F}$ more than one node can simultaneously run out of its battery energy. So, $F_{s}(\widehat{T F F})$ gives us an idea about the percentage of energy balancing at this instance. Under the network configuration described above (Table III), the simulation results are presented in Table IV. It is clear that the use of UWB physical layer can significantly increase the network lifespan, given that a UWB transceiver consumes less energy than classical transceivers as shown in Table I. We note that for both physical layers, the estimation of the fraction of surviving nodes in the network $F_{s}(\widehat{T F F})$ is the same given that this measure does not depend on physical layer but on MAC layer's specifications (duty cycle, resource allocation policy, etc.).

TABLE IV

NETWORK LIFETIME

\begin{tabular}{c|c|c|c}
\hline \hline $\begin{array}{c}\text { Protocol } \\
1: U W B P H Y \\
2: 2.4 P H Y\end{array}$ & $(y / m / d / h / m / s e c)$ & $F_{s}(\widehat{T F F})$ & $\begin{array}{c}\widehat{M E T} \\
(y / m / d / h / m / s e c\end{array}$ \\
\hline \hline$P M C M T P^{1}$ & $-/ 1 / 2 / 7 / 53 / 26$ & $64 \%$ & $1 / 3 / 4 / 6 / 17 / 36$ \\
\hline$P M C M T P^{2}$ & $-/-/ 17 /-/ 20 / 9$ & $64 \%$ & $-/ 5 / 12 / 15 / 20 / 57$ \\
\hline \hline
\end{tabular}

3) Power efficiency: We compare the power efficiency of PMCMTP with MCMAC. According to the result presented in Figure 14, we discover that the power efficiency performance of PMCMTP is much better than that of MCMAC. On the one hand, not only PMCMTP allows parallel communications inside an active PAN but it also allows several active PANs to communicate simultaneously which ensures a considerable throughput increase, on the other hand, given that PMCMTP allocates variable time slots per resource request which can minimize overhead and energy consumption.

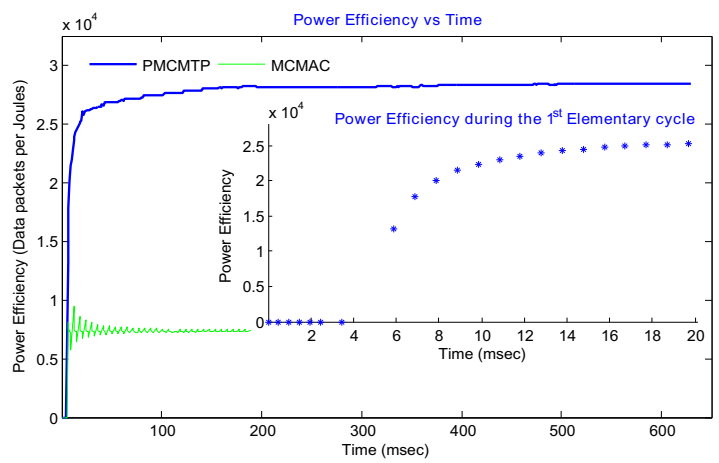

Fig. 14. Power Efficiency

\section{CONCLUSION AND FUTURE WORK}

In this paper, we have presented and evaluated PMCMTP, a multi-channel multi-time slot MAC protocol for dense and large-scale WSNs with QoS support. Given that WSNs are power-constrained, the energy efficiency represents the main goal in the proposed schemes design. According to the network configuration and the available spectrum resource, UWBCAS [18] efficiently shares the UWB spectrum between active PANs then, inside each active PAN, PMCMTP tries to efficiently assign available time slots per channel in response to received resource requests. PMCMTP's performance has been evaluated through a set of simulations, and the experimental results show that our protocol exhibits prominent ability to ensure energy saving and power efficiency. However, in order to ensure a better compromise between energy efficiency, QoS guarantees and resource (spectral and temporal) utility enhancement, it is important to evaluate the impact of lower and higher layers on the PMCMTP. In this context, we propose to jointly deal with duty cycle scheduling, multiconstrained QoS routing and resource allocation problems via the collaboration of all the network layers. The proposition of the suitable cross-layer design for real-time WSNs will be the challenge that we will tackle in next work.

\section{REFERENCES}

[1] X. Wang and T. Berger, "Spatial channel reuse in wireless sensor networks", Springer, vol 14, iss 2, pp. 133-146, March 2008.

2] CC2420 datasheet, inst.eecs.berkeley.edu/ cs150/Documents/CC2420.pdf, June 2010

[3] J. Ben Slimane, Y.Q. Song, and A. Koubâa, "A Prioritized MultiChannel Multi-Time slot MAC Protocol For Large-Scale Wireless Sensor Networks", The First International Conference on Communications and Networking, 2009, Hammamet Tunisia.

[4] IEEE 802.15.4a Standard Part 15.4: IEEE Standard for Information Technology, Amendment to IEEE Std 802.15.4-2006, 2007. 
[5] G. Zhou, C. Huang, T. Yan, T. He, and J. Stankovic, "MMSN: multifrequency media access control for wireless sensor networks", INFOCOM 2006. $25^{t h}$ IEEE International Conference on Computer Communications Proceedings, April 2006, pp. 1-13.

[6] Y. Kim, H. Shin, and H. Cha, "Y-MAC: An Energy-Efficient Multichannel MAC Protocol for Dense Wireless Sensor Networks", International Conference on Information Processing in Sensor Networks, 2008, pp. 53-63.

[7] O.D. Incel, S. Dulman, and P. Jansen, "Multi-channel Support for Dense Wireless Sensor Networking", In Proceedings of the First European Conference on Smart Sensing and Context, EuroSSC 2006, Enschede, the Netherlands, pp. 25-27.

[8] R.E. Cagley, S.A. McNally, and M.R. Wiatt, "Dynamic channel allocation for dynamic spectrum use in wireless sensor networks" Military Communications Conference, October 2006, pp. 1-5.

[9] H. So, W. Walrand, and J. Jeonghoon, "McMAC: a parallel rendezvous multi-channel MAC protocol", IEEE Wireless Communications and Networking Conference, March 2007, pp. 334-339.

[10] Technical overview of time synchronized mesh protocol (tsmp). TSMPWhite Paper, http://www.dustnetworks.com/, 2006.

[11] X. Chen, P. Han, Q. He, S. Tu, and Z. Chen, "A multi-channel MAC protocol for wireless sensor networks", The $16^{\text {th }}$ IEEE International Conference on Computer and Information, 2006, pp. 224-229.

[12] A. Rangnekar and K.M. Sivalingam, "Multiple Channel Scheduling in UWB based IEEE 802.15.3 Networks", Proceedings of the First International Conference on Broadband Networks, October 2004, pp. 406415

[13] R. Prakash, N. Shivaratri, and M. Singhal, "Distributed Dynamic Channel Allocation for Mobile Computing", In Proceedings of the $14^{\text {th }}$ ACM Symposium on Principles of Distributed Computing, August 1995.

[14] J. Ben Slimane, Y.Q. Song, A. Koubâa, and M. Frikha, "A ThreeTiered Architecture for Large-Scale Wireless Hospital Sensor Networks", the International Workshop on Mobilizing Health Information to Support Healthcare-Related Knowledge Work - MobiHealthInf 2009, pp 20-31.

[15] J. Jemai, R. Piesiewicz, and T. Kurner, "Calibration of an indoor radio propagation prediction model at $2.4 \mathrm{GHz}$ by measurements of the IEEE 802.11b preamble", IEEE $61^{\text {st }}$ Vehicular Technology Conference, Spring. 2005, vol.1, pp. 111-115.

[16] A. Koubâa, A. Cunha, and M. Alves, "A Time Division Beacon Scheduling Mechanism for IEEE 802.15.4/Zigbee Cluster-Tree Wireless Sensor Networks", The $19^{t h}$ Euromicro Conference on Real-Time Systems (ECRTS 2007), Pisa(Italy), July 2007.

[17] A. Koubâa, A. Cunha, M. Alves, and E. Tovar, "TDBS: a time division beacon scheduling mechanism for ZigBee cluster-tree wireless sensor networks", Real-Time Systems Journal, October 2008, vol.40, pp. 321354.

[18] J. Ben Slimane, Y.Q. Song, A. Koubâa, and M. Frikha, "Allocation of control and data channels for Large-Scale Wireless Sensor Networks", Technical report, 2008, http://hal.inria.fr/inria-00322584/fr/.

[19] P. Pedreiras, P. Gai, L. Almeida, and G.C. Buttazzo, "FTT-Ethernet A Flexible Real-Time Communication Protocol That Supports Dynamic QoS Management on Ethernet-Based Systems", IEEE Transactions on Industrial Informatics, 2005, vol.1, iss.3, pp. 162-172.

[20] W. Xin, R. Yong, Z. Jun, G. Zihua, and R. Yao, "Energy efficient transmission protocol for UWB WPAN", The $60^{\text {th }}$ IEEE Vehicular Technology Conference, 2004, vol.7, pp. 5292-5296.

[21] Y. Zheng, Y. Tong, J. Yan, Y.P. Xu, and W.G. Yeoh, "A low power noncoherent CMOS UWB transceiver ICs", IEEE Radio Frequency integrated Circuits (RFIC) Symposium, 2005, pp. 347-350.

[22] M.C. Ha, Y.J. Park, and Y.S. Eo, ”A 3-5 GHz Non-Coherent IR-UWB Receiver", Journal of Semiconducteur Technology and Science, 2008 , vol.8, no. 4 , pp. $277-281$.

[23] L. Weinan, H. Yumei, and H. Zhiliang, "A low power $35 \mathrm{GHz}$ CMOS UWB receiver front-end", Journal of Semiconductors, March 2009 , vol.30, no.3, pp. $1-5$.

[24] C. Sandner, S. Derksen, and D. Draxelmayr, "A WiMedia/MBOACompliant CMOS RF Transceiver for UWB", IEEE Journal of Solid-State Circuits, 2006, vol.41, iss.12, pp. 2787-2794.

[25] S. Lee, J. Bergervoet, and K. Harish, "A broadband receive chain in 65nm CMOS", International Journal of Electronics, 2008, vol.95, iss.4 pp. 305312 .

[26] M. Shen, T. Koivisto, and T. Peltonen, "UWB radio module design for wireless sensor networks", The $23^{\text {rd }}$ NORCHIP Conference, 2005, pp. 184-187.
[27] J.H. Chang and L. Tassiulas, "Energy conserving routing in wireless ad-hoc networks", INFOCOM, March 2000, vol.1, pp. 22-31.

[28] R. Wattenhofer, L. Li, P. Bahl, and Y Wang, "Distributed topology control for power efficient operation in multihop wireless ad hoc networks", IEEE INFOCOM, 2001, vol.3, pp. 1388-1397.

[29] J.W. Zhang, Y.Y. Ji, J.J. Zhang, and C.L. Yu, "A Weighted Clustering Algorithm Based Routing Protocol in Wireless Sensor Networks", ISECS International Colloquium on Computing, Communication, Control, and Management, August 2008, pp. 599-602.

[30] T. Zheng, S. Radhakrishnan, and V. Sarangan, "PMAC: an adaptive energy-efficient MAC protocol for wireless sensor networks", Proceedings of the $19^{t h}$ IEEE International Parallel and Distributed Processing Symposium 2005, vol.13, pp. 237-245.

[31] http://www.eclipse.org/, June 2010.

[32] http://www.isis.vanderbilt.edu/projects/nest/prowler/, June 2010. 